BBA 65600

\title{
THE STEADY-STATE RELAXATION TIME AND THE PRODUCT AT EQUILIBRIUM
}

DALE M. MUELLER, KEITH S. HENLEY, LESLIE U. HENDELMAN AND JORGE J. GUMUCIO

Department of Internal Medicine, Section of Gastroenterology, The University of Michigan Medical School, Ann Arbor, Mich. (U.S.A.)

(Received August ist, I966)

(Revised manuscript received February 2nd, 1967)

SUMMARY

I. According to Bücher and his co-workers, the steady-state relaxation time (i.e. the reciprocal of the first-order process by which an enzyme reaction reaches equilibrium) determines, in part, the apparent metabolic flow rate through a given enzymatic step in vivo.

2. A new method for the determination of the kinetic constants required for the calculation of the apparent metabolic flow rates according to BücHER, is developed. The method is based on the measured concentrations of substrate or product in the course of incubation, and has been adapted for use by a digital computer.

3. The relevance of the data thus obtained to the calculated metabolic flow rates are briefly discussed.

INTRODUCTION

It has been suggested by BÜCHER AND RüSSMAN ${ }^{1}$ that the apparent metabolic flow rate through a two-partner reaction of the form $A \rightleftharpoons P$ near equilibrium can be calculated from the following formula:

$$
V_{\mathrm{st}}=\frac{\left(V_{1}\right)_{\mathrm{tissue}}}{\left(\tau_{\mathrm{ss}}\right.} \frac{P\left(K_{\mathrm{app}}-\Gamma\right)}{\left.V_{1}\right)} \frac{K_{\mathrm{app}}(\mathrm{I}+\Gamma)}{\left.K_{\mathrm{s}}+\Gamma\right)}
$$

where $V_{\text {st }}$ is the metabolic flow rate (in terms of unit mass per weight per unit time), $\left(V_{1}\right)_{\text {tissue }}$ is the maximal activity of the enzyme measured in the tissue, $\bar{P}$ is the concentration of the product at equilibrium ${ }^{2}, K_{\text {app }}$ the equilibrium constant, and $\Gamma$ an expression based on the deviation of the substrates from equilibrium. $\tau_{\mathrm{ss}}$ (see refs. 3,4$)$ is the steady-state relaxation time (i.e. the time taken for the deviation from equilibrium to decrease by one e-th part of its original value), and $V_{1}$ the maximal activity of the enzyme used for the determination of the steady-state relaxation time 
in vitro. Determinations of the apparent metabolic flow rates are made possible by the demonstration $^{1}$ that $\left(\tau_{\mathrm{ss}} V_{1}\right)$ is related to $\bar{P}$ by a linear equation of the form:

$$
\tau_{\mathrm{ss}} V_{1}=\alpha \bar{P}+\beta
$$

where $\alpha$ and $\beta$ are the slope and intercept respectively of the linear regression of $\left(\tau_{\mathrm{ss}} V_{1}\right)$ on $P$.

The development of Eqn. I is based on the assumptions ${ }^{1}$ that (I) Equilibrium is approached by a first-order process. (2) Michaelis-Haldane kinetics apply in vivo as well as in vitro regardless of the much greater concentration of the enzyme in vivo compared with that used in vitro, and on the additional assumption. (3) That the deviation of the substrate from equilibrium $(\triangle A)$ is small. The latter is used by BücHER AND RüSSMAN ${ }^{1}$ in the development of their Eqn. 8 which postulates that

$$
\Delta A\left(\frac{\mathrm{I}}{K_{\mathrm{a}}}-\frac{\mathrm{I}}{K_{\mathrm{p}}}\right) \approx 0
$$

where $K_{\mathbf{a}}$ and $K_{\mathrm{p}}$ are the Michaelis constants of substrate and product, respectively. Furthermore, in the experimental determination of the constants for Eqn. 2 certain difficulties may arise:

(I) If $P_{j}$ is the concentration of the product at any time $t_{j}$, the calculation of the steady-state relaxation time depends on $\ln \left(\bar{P}-P_{j}\right)$. If $P$ is only slightly larger than $P_{j}$, minimal errors in reading the optional density result in significant changes in the calculated relaxation times.

(2) Two lines, each representing approximately first-order kinetics may be obtained from the same set of data. These lines are characterized by slightly different slopes and therefore different relaxation times.

As first-order kinetics may be obeyed even for quite large deviations from equilibrium calculations of the metabolic flow rate would be feasible for a wider range of metabolite concentration if the conditions of Eqn. 3 were not obligatory (i.e. if no stipulations were made as to the magnitude of $\Delta A$ and if $K_{\mathbf{a}} \neq K_{\mathrm{p}}$ ).

In this paper a method for the calculation of Eqn. 2 is developed which is independent of Assumption 3, and which attempts to overcome some of the experimental difficulties outlined above. This method will be exemplified by the phosphopyruvate hydratase (2-phospho-D-glycerate hydrolase, EC 4.2.I.II) reaction.

\section{METHODS}

\section{Experimental}

Materials: substrates, co-factors and enzymes were purchased from Boehringer Mannheim Inc.

Phosphopyruvate hydratase was prepared as follows: approx. $50 \mathrm{~g}$ of human liver obtained at autopsy were homogenized in $0.25 \mathrm{M}$ sucrose using a Potter Elvehjem homogenizer with a teflon pestle. For the preparation of the sucrose medium and throughout the preparative procedure, double-distilled water made I mM in EDTA (disodium salt) was used. The final volume was made to $250 \mathrm{ml}$ and the homogenate centrifuged at Io ooo $\times \mathrm{g}$. The $\mathrm{pH}$ of the supernatant was then adjusted to 5.0 with a few drops of I $\mathrm{M} \mathrm{HCl}$ and the concentration of protein ${ }^{5}$ adjusted to $2.2 \mathrm{mg} / \mathrm{ml}$. The material was then warmed to $37^{\circ}$ and the $0.65 \%$ to $0.75 \%\left(\mathrm{NH}_{4}\right)_{2} \mathrm{SO}_{4}$ precipitate 
collected. This precipitate was resuspended in o.I $\mathrm{M}\left(\mathrm{NH}_{4}\right)_{2} \mathrm{SO}_{4}$. Any trace of pigment remaining was readily removed by extraction with a small amount of $\mathrm{CHCl}_{3}$ and without loss of enzyme activity. Except where otherwise stated, all procedures were carried out at $0-4^{\circ}$. The resultant preparation contained phosphopyruvate hydratase and no other enzymes capable of acting on either 2-phosphoglycerate or phosphoenolpyruvate in the absence of added co-factors. When kept in the cold, the preparation lost about $5 \%$ of its activity per day.

For the kinetic studies a suitably dilute sample of the enzyme preparation was incubated with a known concentration of 2-phosphoglycerate in $0.2 \mathrm{M}$ Tris ( $\mathrm{pH} 7.2$ ) at $37^{\circ}$ in a Beckman DU spectrophotometer. The reaction was initiated by the addition of $\mathrm{MgCl}_{2}$ in $3.3 \mathrm{mM}$ final concentration, and the formation of phosphoenolpyruvate recorded at I-min intervals at $240 \mathrm{~m} \mu$ until no further change in absorbance occurred (i.e. until equilibrium had been reached).

The maximal activity of the phosphopyruvate hydratase preparation in the forward direction was measured on the basis of the method described by CzOK AND ECKERT $^{6}$ using lactate dehydrogenase (L-lactate NAD oxidoreductase, EC I.I.I.27) as the indicator for the forward reaction and glyceraldehyde phosphate dehydrogenase (D-glyceraldehyde-3-phosphate NAD oxidoreductase (phosphorylating) EC I.2.I.I2) for the reverse reaction.

The apparent equilibrium constant was determined at $\mathrm{pH} 7.2$ and $37^{\circ}$, and in the Tris buffer using commercial phosphopyruvate hydratase. Following the addition of $\mathrm{MgCl}_{2}$ and either 2-phosphoglycerate or phosphoenolpyruvate, incubation was continued for $30 \mathrm{~min}$. Samples were then taken at 5 -min intervals for up to $45 \mathrm{~min}$, inactivated in about 5 vol. of chilled $6 \mathrm{M} \mathrm{HClO}_{4}$, neutralized and centrifuged. The ratio of phosphoenolpyruvate to 2-phosphoglycerate in the neutralized extract was then determined ${ }^{6}$.

\section{Mathematical}

The calculations depend on the following principles:

(a) The data obtained in the course of incubation of enzyme with substrate are fitted to the integrated form of the Michaelis-Menten equation for a two-partner reaction expressing the concentration of the product formed as a function of time.

(b) A series of such equations were obtained in which the initial substrate concentrations and the enzyme activities were varied independently. Each of these was used for the calculation of $\tau_{\mathrm{ss}}$ and the product of $\tau_{\mathrm{ss}}$ and $V_{1}$ was then plotted against $P$ thus obtaining the regression line given by Eqn. 2 .

If $E$ is the concentration of the enzyme, $A$ the concentration of the substrate and $P$ the concentration of the product then, near equilibrium, when both substrate and product are present, the reaction may be formulated as follows:

$E+A \rightleftharpoons E A \rightleftharpoons E P \rightleftharpoons E+P$

The steady-state velocity is given $b^{7}$

$$
-\frac{\mathrm{d} A}{\mathrm{~d} t}=\frac{\mathrm{d} P}{\mathrm{~d} t}=\frac{V_{\mathrm{a}} A / K_{\mathrm{a}}-V_{\mathrm{p}} P / K_{\mathrm{p}}}{\mathrm{I}+\frac{A}{K_{\mathrm{a}}}+\frac{P}{K_{\mathrm{p}}}}
$$

where $V_{\mathrm{a}}$ and $V_{\mathrm{p}}$ are the maximal velocities in the direction $A$ to $P$ and $P$ to $A$, 
respectively, and $K_{\mathrm{a}}$ and $K_{\mathrm{p}}$ are their respective substrate constants. Integration of this equation ${ }^{7}$ yields:

$t\left[\frac{V_{\mathrm{a}}}{K_{\mathrm{a}}}+\frac{V_{\mathrm{p}}}{K_{\mathrm{p}}}\right]=\left[\mathrm{I}+\frac{A_{0}}{K_{\mathrm{p}}}+\frac{\left(\frac{\mathrm{I}}{K_{\mathrm{a}}}-\frac{\mathrm{I}}{K_{\mathrm{p}}}\right)}{\frac{V_{\mathrm{a}}}{K_{\mathrm{a}}}+\frac{V_{\mathrm{p}}}{K_{\mathrm{p}}}} \frac{V_{\mathrm{p}}}{K_{\mathrm{p}}} A_{\mathrm{v}}\right] \ln \frac{A_{\mathrm{o}}-A}{A-A}+\left[\frac{\mathrm{I}}{K_{\mathrm{a}}}-\frac{\mathrm{I}}{K_{\mathrm{p}}}\right]\left(A_{\mathrm{o}}-A\right)$

where $A_{0}$ is the initial substrate concentration (i.e. the substrate concentration when $P=0$ ). The concentrations of the metabolites $A$ and $P$ at various times $t$ are obtained from the experimental data. $\bar{A}$ then is the concentration of the substrate at equilibrium. A mathematical expression which permits the experimental data to be fitted to Eqn. 4 was developed as follows:

In the absence of interfering reactions, the sum of substrate and product is constant

$$
\begin{aligned}
& A=A_{0}-\bar{P} \\
& A=A_{0}-P
\end{aligned}
$$

substituting these equations into Eqn. 4 and simplifying:

$$
t=\left[\frac{K_{\mathrm{a}} K_{\mathrm{p}}}{\overline{V_{\mathrm{a}} K_{\mathrm{p}}-K_{\mathrm{a}} V_{\mathrm{p}}}}\right]\left[\mathrm{r}+\frac{A_{\mathrm{a}}\left(V_{\mathrm{a}}+V_{\mathrm{p}}\right)}{V_{\mathrm{a}} K_{\mathrm{p}}+K_{\mathrm{a}} V_{\mathrm{p}}}\right] \ln \frac{\bar{P}}{\bar{P}-P}+\left[\frac{K_{\mathrm{p}}-K_{\mathrm{a}}}{V_{\mathrm{a}} K_{\mathrm{p}}-K_{\mathrm{a}} V_{\mathrm{p}}}\right] P
$$

Using the well-known HALDANE-ALBERTY relationship ${ }^{8}$

$$
K_{\mathrm{app}}=\frac{V_{\mathrm{a}} K_{\mathrm{p}}}{V_{\mathrm{p}} K_{\mathrm{a}}}=\frac{\bar{P}}{\bar{A}}=\frac{\bar{P}}{A_{0}-\bar{P}}
$$

or

$$
K_{\mathrm{p}}=\frac{\bar{P} V_{\mathrm{p}}}{\left(A_{\mathbf{0}}-\overline{\bar{P}}\right) V_{\mathrm{a}}} K_{\mathrm{a}}
$$

and substituting into Eqn. 5 and simplifying:

$$
t=\left[\frac{\bar{P}}{A_{0} V_{\mathrm{a}}}\right]\left[K_{\mathrm{a}}+\frac{\left(V_{\mathrm{a}}+V_{\mathrm{p}}\right)\left(A_{0}-\bar{P}\right)}{V_{\mathrm{p}}}\right] \ln \frac{\bar{P}}{\bar{P}-\bar{P}}+\left[\frac{P\left(V_{\mathrm{a}}+V_{\mathrm{p}}\right)-A_{\mathrm{a}} V_{\mathrm{a}}}{A_{\mathrm{a}} V_{\mathrm{a}} V_{\mathrm{p}}}\right] P
$$

The only unknown parameter in Eqn. 8 is $K_{\mathrm{a}}$ because both $V_{\mathrm{a}}$ and $V_{\mathrm{p}}$ have been measured experimentally. To obtain the maximum likelihood estimate of this value, the least-squares regression criterion was employed as follows:

A function $G\left(K_{\mathrm{a}}\right)$ was defined such that

$$
G\left(K_{\mathrm{a}}\right)=\sum_{j=\mathrm{I}}^{n}\left(t_{\mathrm{o}_{j}}-t_{\mathrm{p}_{j}}\right)^{2}
$$

where $j$ is an index on the data points, $t_{\mathrm{o}}$ identifies the observed data, and $t_{\mathrm{p}}$ the predicted points. The value of $K_{\mathrm{a}}$ for which $G\left(K_{\mathrm{a}}\right)$ is a minimum is given by $\frac{\mathrm{d} G\left(K_{\mathrm{a}}\right)}{\mathrm{d} t}=\mathrm{o}$

Substituting the expression on the right of Eqn. 8 for $t_{\mathrm{p}_{j}}$ into Eqn. 9 differentiating, simplifying and solving for $K_{\mathrm{a}}$ we obtain:

$$
K_{\mathrm{a}}=\frac{A_{\mathrm{o}} V_{\mathrm{a}} V_{\mathrm{p}} \sum_{j} t_{j} \ln \frac{P}{\bar{P}-P_{j}}-P\left(V_{\mathrm{a}}+V_{\mathrm{p}}\right)\left(A_{\mathrm{o}}-\bar{P}\right) \sum_{j}^{2} \ln \frac{\bar{P}}{\bar{P}-P_{j}}-\left[\bar{P}\left(V_{\mathrm{a}}+V_{\mathrm{p}}\right)-A_{0} V_{\mathrm{a}}\right] \sum_{j} P_{j} \ln \frac{P}{\bar{P}-P_{j}}}{V_{\mathrm{p}} \bar{P} \Sigma \ln ^{2} \frac{\bar{P}}{\bar{P}-P_{j}}}
$$


It is then possible to calculate $K_{\mathrm{p}}$ from Eqn. Io and Eqn. 7. These values are then substituted into Eqn. 4 .

Eqn. 4 is then used for the calculation of the steady-state relaxation time, $\tau_{\mathrm{ss}}$, as follows:

By definition ${ }^{1} \tau_{\mathrm{ss}}$ is that interval of time $\left(t_{2}-t_{1}\right)$ for which the corresponding value $\left(P_{2}-P_{1}\right)$ represents an increase of $P_{1}$ by one e-th of its value, i.e.

$$
P_{2}=\left(P_{1}+\frac{P_{1}}{\mathrm{e}}\right)
$$

First-order kinetics on which these calculations are based are best met near equilibrium (i.e. when $P_{2}=\bar{P}$ ). However, as $P \rightarrow P, t \rightarrow \infty$, and for this reason a constant $c$ is selected such that $P_{2}=c P$ where $c$ is near unity (e.g. 0.99). If $P_{2}=c P$ then from Eqn. II

and

$$
c \bar{P}=\left(\mathrm{I}+\frac{\mathrm{I}}{\mathrm{e}}\right) P_{1}
$$

$$
P_{1}=\left(\frac{\mathrm{e}}{\mathrm{e}+\mathrm{I}}\right) c \bar{P}
$$

Therefore

$$
\tau_{\mathrm{ss}} \text { (in minutes) }=\left[\iota(c \bar{P})-\iota\left(c \frac{\mathrm{e}}{\mathrm{e}+\mathrm{I}} \bar{P}\right)\right]
$$

Substituting into Eqn. 4, multiplying by $V_{1}$, factoring and collecting terms:

$$
\tau_{\mathrm{ss}} V_{1}=V_{1}\left\{\left[\frac{K_{\mathrm{a}} K_{\mathrm{p}}}{V_{\mathrm{a}} K_{\mathrm{p}}+K_{\mathrm{a}} V_{\mathrm{p}}}\right]\left[\mathrm{I}+\frac{A_{0}\left(V_{\mathrm{a}}+K_{\mathrm{p}}\right)}{V_{\mathrm{a}} K_{\mathrm{p}}+K_{\mathrm{a}} V_{\mathrm{p}}}\right] \ln \left[\frac{\mathrm{e}(\mathrm{I}-c)+\mathrm{I}}{(\mathrm{e}+\mathrm{r})(\mathrm{I}-c)}\right]+\frac{K_{\mathrm{p}}-K_{\mathrm{a}}}{V_{\mathrm{a}} K_{\mathrm{p}}-K_{\mathrm{a}} V_{\mathrm{p}}} c \frac{\mathrm{I}}{\mathrm{e}+\mathrm{I}} P\right\}
$$

The relationship between $\left(\tau_{\mathrm{ss}} V_{\mathbf{1}}\right)$ and $\bar{P}$ should be linear and of the form given by Eqn. 2. From the maximum likelihood estimates of the coefficient and intercept of linear regression equations, $\alpha$ and $\beta$ are given be Eqns. I3 and I4.

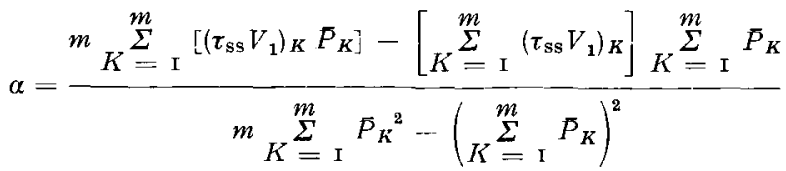

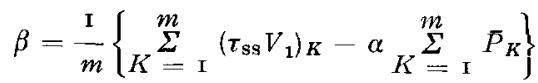

where $k$ is an index on the data points for $m$ number of experiments of the type described earlier.

Eqns. IO, I2-I4 are readily translated into suitable computer language. (A program written in the FORTRAN II compiler language and suitable for an IBM 7090 digital computer may be obtained from the authors upon request.)

\section{RESULTS AND DISCUSSION}

In a mean of five experiments $K_{\text {app }}$ was found to be 5.I3, fairly close to the value of 4.8 which can be calculated from the data of BÜcHER AND RÜSSMAN ${ }^{1}$ (op. cit. Fig. 4). The ratio of $V_{\mathrm{a}} / V_{\mathrm{p}}$ was 3.72 , somewhat higher than that given for ascites tumor cells 9 
The experimental data fitted the curve given by Eqn. 4 with a standard error of the mean of $10.7 \%$ based on 22 experiments using enzyme prepared from one specimen of human liver. The linear relationship between $\tau_{\mathrm{ss}} V_{1}$ and $\bar{P}$ was computed from these experiments and is illustrated in Fig. I. It is evident that the data fit a linear regression line which would be anticipated if Eqn. 2 were valid, and with sufficient precision to be used as a basis for the computation of metabolic flow rates.

As will be seen from inspection of Eqns. I and 2 the apparent metabolic flow rate is inversely related to $\alpha$, the slope of Eqn. 2. The slope of the regression line is

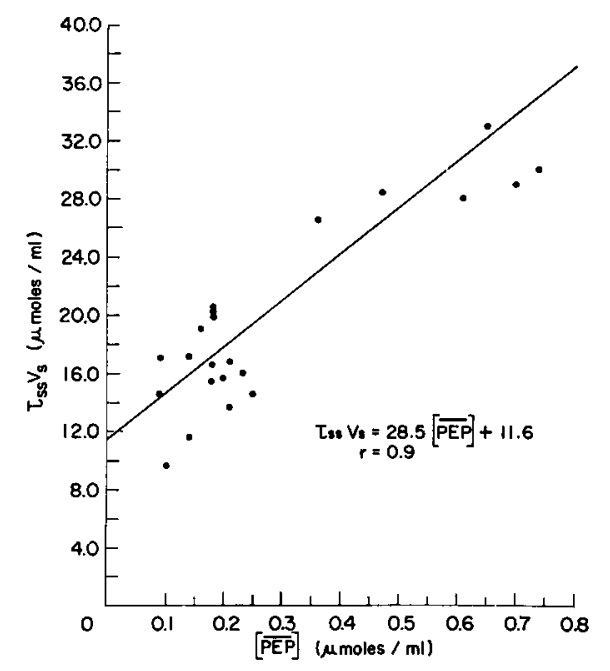

Fig. I. Plot of $\left(\tau_{\mathrm{ss}} V_{1}\right)$ against $P$. Enolase from normal human liver. PEP stands for phosphoenolpyruvate.

higher and the calculated metabolic flow rate therefore lower than in the experiments of BÜCHER AND RÜSSMAN ${ }^{1}$ which were based on enolase derived from muscle. Whether this difference is due to the more sluggish rate of glycolysis which characterizes liver compared with muscle, due to the differences in the mathematical treatment of the data, or both remains to be determined.

The procedure outlined in this paper clearly could be extended to other twopartner reactions such as the phosphoglucose isomerase (D-glucose-6-phosphate ketol isomerase, $\mathrm{EC}$ 5.3.I.9) reaction and studies to explore this possibility are in progress.

\section{ACKNOWLEDGEMENTS}

This study has been supported by grant AM 0736I and Research Career Development Award I403o of the National Institutes of Health to K.S.H. Our thanks are due to Dr. D. Goldsack, Dr. E. A. Napier, Jr. and Dr. G. Palmer for helpful suggestions and criticisms and to Mr. E. G. LAUGHREY for technical asistance.

\section{REFERENCES}

I Th. Bücher and W. Rüssman, Angew. Chem. Intern. Ed. Engl., 3 (I964) 426. 
2 W. W. Cleland, Biochim. Biophys. Acta, 67 (1963) Io 4.

3 M. EIGEN, Discussions Faraday Soc., I7 (1954) I94.

4 G. G. Hammes and E. A. Alberty, J. Am. Chem. Soc., 82 (1960) I 564.

5 O. H. Lowry, N. J. Rosebrough, A. L. Farr and R. J. Randall, J. Biol. Chem., i93 (I95I) 265.

6 R. Czok And L. Eckert, in H. U. Bergmeyer, Methods of Enzymatic Analysis, Academic Press, New York, 1963, p. 223.

7 R. A. Alberty, Advan. Enzymol., I7 (I956) 2.

8 J. B. S. Haldane, Enzymes, Longmans, Green and Co., London, I930, p. 80.

9 B. Hess, Funktionelle und Morphologische Organisation der Zelle, Springer, Berlin, I963, p. I63.

Biochim. Biophys. Acta, 139 (1967) 231-237 